\title{
The Relationship Between Health Belief and Breast Self-examination Among Iranian University Students
}

\author{
Elnaz Asghari ${ }^{1}$, Mina Nahamin ${ }^{1}$, Mehrnoosh Khoshtarash ${ }^{1}$, Atefe Ghanbari ${ }^{2}$, Naser Parizad ${ }^{1}$, Nader \\ Mahdavi $^{3}$, Zoleikha Asgarlo ${ }^{*}$
}

\begin{abstract}
Objectives: Breast cancer is a major health problem. Breast self-examination (BSE) is a simple method for breast cancer screening. The health belief model (HBM) is a model for conventionalizing and management of health-related behaviors such as breast cancer screening. The objective of this study was to determine the relationship between HBM and BSE among Iranian university students. Materials and Methods: This was a cross-sectional study conducted on 247 medical and paramedical students of Guilan University of Medical Sciences in 2010. Data was collected using Champion's Health Belief Model Scale (CHBMS) for breast cancer screening whose validity and reliability have been verified in previous studies. Data was analyzed using descriptive and inferential statistics. Results: The average age of samples was $21.76 \pm 2.6$ years. Students with a positive family history of breast cancer performed BSE more frequently $(9.2 \%$ compared with $82.2 \%, P<0.05)$. The mean score of Health Belief Questionnaire was low for the majority of samples $(61.94 \%, \mathrm{n}=153)$. Students' health beliefs had a statistically significant relationship with performing BSE $(P<0.05)$.

Conclusion: Since medical and paramedical students are regarded as role models of healthy behaviors, it is critical to have an awareness of their health beliefs on BSE. In addition, positive behavior and attitude towards BSE enables students to perform breast cancer screening tests more effectively. According to the results, it is necessary to improve students' knowledge of and attitude towards BSE by training programs.

Keywords: Breast cancer, Breast self-examination, Health belief
\end{abstract}

\section{Introduction}

Breast cancer, as a major health concern in females, is the most common type of cancer across the world (1). The incidence of breast cancer varies from the United States of America, with the highest incidence, to some Asian countries in which the incidence of this disease is the lowest. However, this rate has increased in these countries due to lifestyle changes (2). In Turkey, for instance, breast cancer is the most common type of cancer and the second cancer-related cause of death in females with an incidence rate of $35.5 \%$ per 100000 women. Although breast cancer used to be among the least common types of cancer, it is now one of the most prevalent ones due to "poor" lifestyles. Although no precise statistical data exists on breast cancer in Iran, unofficial reports show that it constitutes $21.4 \%$ of all reported cases of cancer in the country. The crude incidence rate of this disease in Iran is 4 in every 100000 women (2). Therefore, without any specific prevention strategy, the most effective method of increasing the survival rate for this cancer is early diagnosis, a simple way of which is breast self-examination (BSE).

BSE is a simple, safe, affordable and non-invasive method for breast cancer screening (3). Despite such advantages and the well-known efficacy of BSE, studies report that the monthly performance of BSE is lower than 15\% (4,5). BSE practices in Iran are not satisfactory either (2). It appears that such factors as awareness of breast cancer, education level, family history of breast cancer, lack of physician recommendation and poor health beliefs and attitudes concerning this disease are correlated with performing BSE and mammography (6). Health beliefs usually account for individual health-related behaviors and knowledge. Health belief model (HBM) is a model for the management of health-related behaviors such as breast cancer screening that focuses on individual beliefs and behaviors (3). HBM is a common theory in health education as well, which can explain the relationship between health promotion messages and behaviors (3). Although in some cases, no relationship has been established between health beliefs and screening behaviors (7), some studies have pointed to a positive relationship between health beliefs and BSE (8).

Employees working in the healthcare system are among the best groups to provide necessary and accurate information and training on breast cancer screening to the public. They make regular contacts with them and can identify the most susceptible individuals (3). On the other hand, according to suggestions of other studies, women's opinions on BSE have to be properly understood to promote such health screening behaviors (2). Given the significance of BSE and the educational role of medical and paramedical students, we aimed at determining the relationship between health beliefs and BSE among university students.

\section{Materials and Methods}

This was a cross-sectional study conducted on medical and paramedical students of Guilan University of Medical Sciences 
in 2010. A total of 247 female students, who volunteered to participate in this study, were selected. Studying in one of the fields of medical sciences with a minimum of 1 year of internship experience in a clinical setting was among the inclusion criteria. Sample size was selected based on a similar study (2) with a $95 \%$ $\mathrm{CI}$ and a $3 \%$ accepted margin of error.

The employed questionnaire included items on demographic characteristics such as age, marital status, education level, family history of breast cancer and performing BSE. The Champion's Health Belief Model Scale (CHBMS) was also used. HBM has been primarily used to study related factors of breast cancer screening behaviors such as performing BSE. According to $\mathrm{HBM}$, individuals have to come to the understanding that a situation can potentially lead to serious consequences (the perceived severity and difficulty) and believe that a series of measures can bring about positive outcomes (the perceived benefits). According to this model, it is also necessary to develop an understanding with respect to susceptibility or sensitivity to a specific kind of disease/illness (the perceived sensitivity). People have concluded that barriers against taking appropriate measures are overshadowed by their advantages. This model also evaluates the perceived self-efficacy (what an individual imagines to be capable of), which reflects patients' perception of their capabilities in performing BSE accurately. The validity and reliability of this scale have been demonstrated in a number of Iranian studies $(2,8)$. Previous Cronbach alpha for the CHBMS varied from 0.79 to $0.94(2,3,5,8)$.

The latest version of this scale was adopted in this study. The questionnaire included 19 items. All related items to HBM were scored using a 5-point scale. They showed the extent to which they agreed with each item, with higher numbers suggesting higher levels of agreement with that item. Respondents' perception of their susceptibility to breast cancer was evaluated with 2 items (e.g. what do you have to say in response to whether the probability of your developing cancer is much lower/higher than a normal woman), $(\alpha=0.64)$. The perceived severity of breast cancer was measured using 4 items (e.g. if I had breast cancer, my life would have completely changed), $(\alpha=0.71)$. The perceived benefits of doing BSE was evaluated using 3 items (e.g. performing regular BSE means that early diagnosis of breast cancer is possible), $(\alpha=0.69)$. Respondents were given an 8 -item list of barriers for doing BSE and were asked to show the extent to which each barrier inhibited regular BSE practices. Five items were used to measure the perceived emotional barriers based on an analysis of the perceived barriers to the regular practice of BSE (for instance, finding BSE emotionally irritating), ( $\alpha=0.82)$. Three items were used to measure the perceived skill barriers (e.g. concern for inability to properly examine my breasts), $(\alpha=0.68)$. The perceived self-efficacy was evaluated using 2 items (e.g. I am confident that I can examine my breasts regularly) $(\alpha=0.75)$. In addition, the questionnaire included a single question to determine the intention to perform BSE (e.g. I intend to do BSE regularly over the next year). Respondents were asked to specify the extent to which they performed BSE practices at the time using 7 categories of answers: rarely/never [0], once a year [1], 3-4 times a year [2], once a month [3], once every two weeks [4], once a week [5] and once a day or more [6]. To examine family history, respondents specified the number of first- and second-degree relatives suffering from breast cancer. They were also classified based on once a month BSE practice [1] or less [0] as well as a range of social-demographic information. According to similar studies $(3,5)$, attitude towards health was classified into two low (poor) and high (good) attitudes towards health.
SPSS 13 was used for the statistical analysis of the data. Normal distribution of data was examined using the Kolmogorov-Smirnov test. Descriptive statistics, including frequency and percentage were used for nominal data and mean \pm standard deviation (SD) was used for continuous data. Chi-square and Spearman correlation tests were used to examine the hypothesis on the relationship between nominal and numerical data, respectively. $P<0.05$ was considered to be statistically significant.

\section{Results}

The mean age of samples was $21.76 \pm 2.6$ years. The distribution of other demographic data is presented in Table 1 . The positive family history of breast cancer was mainly related to their grandmothers $(31.57 \%, \mathrm{n}=6)$. Among those who did not perform BSE regularly, "I do not think about it" $(17.56 \%, \mathrm{n}=39)$, "I do not know" and "I do not have the necessary skill to do it" (14.86\%, $\mathrm{n}=33$ ) constituted the main causes, respectively. Students with a positive family history of breast cancer performed BSE practices more often than their counterparts $(n=16$ out of $19,82.2 \%$, compared with $\mathrm{n}=21$ out of $228,9.2 \%, P<0.05)$.

Students' health beliefs had a statistically significant relationship with performing BSE $(P<0.05)$. Attitude towards health was low for the majority of samples $(n=153,61.94 \%)$. More analyses of HBM are depicted in Table 2. Regular BSE was significantly related to all health belief subscales other than "the perceived severity." In addition, irregular BSE was significantly related to all health belief sub-scales other than "the perceived severity."

\section{Discussion}

Women have to be educated on BSE, which would enable them to diagnose breast cancer at early stages. This study aimed at investigating the relationship between health beliefs and BSE among university students. Results showed that a small percentage $(10.12 \%)$ of subjects performed BSE regularly. Studies on healthcare personnel reports $6 \%-83 \%$ of regular BSE practices (9-11). In general, considerable increase in screening behaviors was observed in previous studies (12). Unfortunately, results from our study pointed to much less frequent regular BSE compared to the general population. This could be accounted for by our young samples who were of less age compared to those of other studies (35-45 years of age), or by little work experience on their part (9-11); since older individuals have seen more cases with breast cancer and according to the HBM, have also found themselves at risk (7).

According to the results, individuals with a positive family history of breast cancer are more likely to do BSE. Moreover, those

Table 1. Socio-Demographic Characteristics of the Respondents

\begin{tabular}{lcc}
\hline Characteristics & $\mathbf{n}$ & $\mathbf{\%}$ \\
\hline Marital status & & \\
$\quad$ Married & 20 & 8.09 \\
$\quad$ Single & 227 & 91.91 \\
Performing BSE & & \\
$\quad$ Yes, Regularly & 25 & 10.12 \\
$\quad$ Yes, Irregularly & 74 & 29.95 \\
No & 148 & 59.91 \\
Clinical work experience & & \\
$\quad$ Yes & 13 & 5.26 \\
$\quad$ No & 234 & 94.73 \\
Family history of breast cancer & & \\
Yes & 19 & 7.69 \\
$\quad$ No & 228 & 99.98 \\
\hline
\end{tabular}


Table 2. The Correlation Between the Health Belief Model (HBM) Variables and Breast Self-examination (BSE)

\begin{tabular}{|c|c|c|c|c|c|c|c|c|c|c|}
\hline & Mean & SD & BSE regular & BSE irregular & INT & SEF & SKL & EMO & BEN & SEV \\
\hline Perceived Susceptibility (SUS) & 3.46 & 0.63 & 0.04 & $0.08^{a}$ & 0.05 & $-0.07 a$ & 0.00 & 0.05 & -0.05 & $0.17^{c}$ \\
\hline Perceived Severity (SEV) & 3.08 & 0.72 & 0.01 & 0.02 & 0.05 & -0.02 & $0.10^{b}$ & $0.16^{c}$ & 0.01 & \\
\hline Perceived Benefits (BEN) & 4.00 & 0.71 & $0.17^{c}$ & $0.26^{c}$ & $0.40^{\mathrm{c}}$ & $0.56^{c}$ & $-0.31^{c}$ & $-0.13^{c}$ & & \\
\hline Perceived Emotional Barriers (EMO) & 1.22 & 0.48 & $-0.24^{c}$ & $-0.23^{c}$ & $-0.36^{c}$ & $-0.24^{c}$ & $0.47^{c}$ & & & \\
\hline Perceived Skill Barriers (SKL) & 1.64 & 0.81 & $-0.35^{c}$ & $-0.39^{c}$ & $-0.43^{c}$ & $-0.51^{c}$ & & & & \\
\hline Self-Efficacy (SEF) & 3.51 & 0.91 & $0.38^{c}$ & $0.38^{c}$ & $0.51^{\mathrm{c}}$ & & & & & \\
\hline Intention (INT) & 4.36 & 0.73 & $0.36^{c}$ & $0.51^{c}$ & & & & & & \\
\hline Doing BSE irregularly & $74^{d}$ & $29.95^{e}$ & $0.44^{c}$ & & & & & & & \\
\hline Doing BSE regularly & $25^{d}$ & $10.12 \mathrm{e}$ & & & & & & & & \\
\hline
\end{tabular}

a $P<0.05,{ }^{b} P<0.01,{ }^{c} P<0.001 ;{ }^{d}$ Number, ePercent.

with higher levels of perceived cancer risk significantly exhibit higher levels of perceived susceptibility. On the other hand, a positive perception of susceptibility to cancer has a significant relationship with doing BSE.

More regular BSE practices have been reported in women with a positive family history of breast cancer (5) which could be due to sufficient awareness of the relationship between genetic factors and breast cancer on their part. Tastan et al reported that subjects with a positive family history of breast cancer have a higher awareness level and knowledge of screening methods (3). It seems that individuals with a positive family history of breast cancer are more inclined to think that they have such risk factors. In other words, students with a positive family history of breast cancer know that they can be at greater risks. Therefore, they try to perform BSE practices in a regular manner to prevent further malignancy. In a study which showed that only $6 \%$ of women did regular BSE practices, it was found that "lack of awareness and deeming BSE unnecessary" was the most common justification for not performing BSE and not receiving medical examination (2).

Other studies have stressed the relationship between offering trainings to subjects and doing BSE (3). Results from another study revealed that offering training on BSE increased its frequency considerably (1). It appears that sufficient information on breast cancer positively affects BSE. Previous studies in Iran revealed that lower levels of BSE practices among women were related to lower levels of education, lack of awareness of cancer and lack of necessary knowledge to perform BSE (2). However, despite the fact that all participants in this study were educated, the level of regular BSE practices was very low. This could be explained by fear of finding a tumor and not by lower awareness levels, as similar studies have demonstrated that one of the major reasons inhibiting women from doing screening tests such as BSE is the fear of discovering a tumor and subsequent surgical operations (3).

In the present study, intention to perform BSE depended on the perceived benefits and barriers which is consistent with the results of Norman and Brian $(P<0.0001)(13)$. On the other hand, the perceived benefits and barriers were related with performing BSE. Consequently, the results of this study are largely consistent with previous applications of HBM in relation with BSE which acknowledged the significant effects of perceived benefits and barriers $(13,14)$. On the other hand, non-significant effects were reported with respect to the perceived severity $(13,15)$; whereas inconsistent results have been yielded on the perceived sensitivity in that it has a significant relationship in some studies (14) and a non-significant relationship in others $(13,15)$.

The results demonstrated that the perceived self-efficacy is related with behavioral trends and doing BSE, which is consistent with previous studies that had reported a significant relationship between the perceived self-efficacy and BSE. Results revealed that individuals with higher levels of health beliefs are less likely not to perform BSE. Similarly, results from previous studies showed that health belief subscales such as health motivation, benefits, barriers and BSE sensitivity are related to doing BSE (8). Other studies demonstrated that a relationship exists between approach, behaviors, beliefs and early diagnosis (16) in that an ideal health attitude results in doing regular BSE practices and consequently to early diagnosis. In a similar vein, some studies have shown that majority of breast tumors are initially detected by patients by touch (17). It should be noted that, based on previous published articles, offering training on breast cancer influences women's health beliefs as well $(12,16,18)$; therefore, it seems that improving students' health beliefs may lead to regular BSE practices.

\section{Limitations}

This study was based on a sample taken from female students in a particular area and thus, cannot be generalized to the general population. The study needs to be repeated with a large more diversified sample and by taking cultural barriers to screening into account.

\section{Conclusion}

The incidence of breast cancer is increasing. Patients suffering from advanced stages of this disease in Iran are relatively younger (about 10 years) than their western counterparts (2). BSE is a simple and effective method to make women aware of breast cancer and encourage them to be examined by physicians in early stages. Approach, behaviors and particularly informed health beliefs on BSE can help the early diagnosis of breast cancer. Results from this study revealed that not only did the selected samples not perform BSE practices, but also they did not provide any training to those admitted by them. Moreover, they lacked sufficient and proper health belief.

The importance of this issue lies in the positive relationship between BSE and health belief. In other words, it appears that BSE behaviors could be more frequently observed by acceptable health beliefs. Therefore, it is necessary to improve BSE and health belief behaviors due to the important role of samples in public health. It seems that more training programs can help students update their knowledge of breast cancer screening to 
promote doing BSE.

There are several implications to the results of this study that promote regular BSE practices in women with a positive family history of breast cancer; particularly those engaged in the healthcare system who need to continue highlighting the positive advantages of regular BSE and the potential barriers that women might experience. Regarding the perceived self-efficacy, the relevant interventions have to be made in order to improve women self-confidence about their ability to perform regular BSE.

\section{Ethical Issues}

Permission to conduct the present study was acquired from the Ethics Committee of Guilan University of Medical Sciences. Informed consents were obtained of all university students after explaining the study objectives and procedure.

\section{Conflict of Interests}

The authors declare no conflict of interests.

\section{Finnacial Support}

The researchers received no financial support or grant from any funding agency in the public and commercial sectors.

\section{Acknowledgments}

Our gratitude goes to all participants, colleagues and dormitory superintendents who helped us during the sampling process.

\section{References}

1. Secginli S, Nahcivan NO. The effectiveness of a nursedelivered breast health promotion program on breast cancer screening behaviours in non-adherent Turkish women: A randomized controlled trial. Int J Nurs Stud. 2011;48(1):2436. doi: 10.1016/j.ijnurstu.2010.05.016.

2. Aghamolaei T, Hasani L, Tavafian SS, Zare S. Improving breast self-examination: an educational intervention based on health belief model. Iran J Cancer Prev. 2011;4(2):82-87.

3. Tastan S, Iyigün E, Kilıc A, Unver V. Health beliefs concerning breast self-examination of nurses in Turkey. Asian Nurs Res. 2011;5(3):151-156. doi:10.1016/j. anr.2011.09.001.

4. Ekici E, Utkualp N. Women instructors behaviors towards breast cancer. J Breast Health. 2007;3:136-197.

5. Karayurt O, Dramal A. Adaptation of Champion's Health Belief Model Scale for Turkish women and evaluation of the selected variables associated with breast self-examination. Cancer Nurs. 2007;30(1):69-77.

6. Kim JH, Menon U. Pre-and postintervention differences in acculturation, knowledge, beliefs, and stages of readiness for mammograms among Korean American women. Oncol Nurs Forum. 2009;36(2):E80-E92. doi:10.1188/09.ONF.
E80-E92.

7. Brunero S, Lamont S. Health behaviour beliefs and physical health risk factors for cardiovascular disease in an outpatient sample of consumers with a severe mental illness: a crosssectional survey. Int J Nurs Stud. 2010;47(6):753-760. doi: 10.1016/j.ijnurstu.2009.11.004.

8. Tavafian SS, Hasani L, Aghamolaei T, Zare S, Gregory D. Prediction of breast self-examination in a sample of Iranian women: an application of the Health Belief Model. BMC Womens Health. 2009;9(1):37. doi:10.1186/1472-6874-9-37

9. Alkhasawneh IM, Akhu-Zaheya LM, Suleiman SM. Jordanian nurses' knowledge and practice of breast selfexamination. J Adv Nurs. 2009;65(2):412-416. doi:10.1111/ j.1365-2648.2008.04891.X.

10. Ibrahim NA, Odusanya OO. Knowledge of risk factors, beliefs and practices of female healthcare professionals towards breast cancer in a tertiary institution in Lagos, Nigeria. BMC Cancer. 2009;9(1):76. doi:10.1186/14712407-9-76.

11. Canbulat N, Uzun Ö. Health beliefs and breast cancer screening behaviors among female health workers in Turkey. Eur J Oncol Nurs. 2008;12(2):148-156. doi:10.1016/j. ejon.200.12.002.

12. Han HR, Lee H, Kim MT, Kim KB. Tailored lay health worker intervention improves breast cancer screening outcomes in non-adherent Korean-American women. Health Education Res. 2009;24(2):318-329. doi:10.1093/ her/cyn021.

13. Norman P, Brain K. An application of an extended health belief model to the prediction of breast selfexamination among women with a family history of breast cancer. Br J Health Psychol. 2005;10(1):1-16. doi: $10.1348 / 135910704 X 24752$.

14. Luszczynska A, Schwarzer R. Planning and selfefficacy in the adoption and maintenance of breast selfexamination: a longitudinal study on self-regulatory cognitions. Psychol Health. 2003;18(1):93-108. doi: $10.1080 / 0887044021000019358$.

15. Avci IA, Kurt H. Health beliefs and mammography rates of Turkish women living in rural areas. J Nurs Scholarsh 2008;40(2):170-175. doi:10.1111/j.1547-5069.2008.00222.x.

16. Avc1 İA. The health beliefs relating to mammography of midwives and nurses. Meme Sağlığı Dergisi. 2007;3(2):4-9.

17. Özmen V. Breast cancer in the world and Turkey. J Breast Health. 2008;4(2):6-12.

18. Gürsoy AA, Ylmaz F, Nural N, et al. A different approach to breast self-examination education: daughters educating mothers creates positive results in Turkey. Cancer Nurs. 2009;32(2):127-34. doi:10.1097/NCC.0b013e3181982d7b.

Copyright $\odot 2016$ The Author(s); This is an open-access article distributed under the terms of the Creative Commons Attribution License (http://creativecommons.org/licenses/by/4.0), which permits unrestricted use, distribution, and reproduction in any medium, provided the original work is properly cited. 